\title{
Energy loss, range, and electron yield comparisons of the CRANGE ion-material interaction code
}

\author{
P. H. Stoltz,${ }^{1, *}$ S. A. Veitzer,${ }^{1}$ R. H. Cohen, ${ }^{2}$ A. W. Molvik, ${ }^{2}$ and J.-L. Vay ${ }^{3}$ \\ ${ }^{1}$ Tech-X Corporation, 5621 Arapahoe Ste. A, Boulder CO 80303 \\ ${ }^{2}$ Lawrence Livermore National Laboratory, \\ P.O. Box 808, Livermore CA 94550 \\ ${ }^{3}$ Lawrence Berkeley National Laboratory, Berkeley, CA 94720
}

(Dated: June 11, 2004 DRAFT)

\begin{abstract}
We present comparisons of the CRANGE code to other well-known codes, SRIM and ASTAR, and to experimental results for ion-material interactions such as energy loss per unit length, ion range, and ion induced electron yield. These ion-material interaction simulations are relevant to the electron cloud effect in heavy ions accelerators for fusion energy and high energy density physics. Presently, the CRANGE algorithms are most accurate at energies above $1.0 \mathrm{MeV} / \mathrm{amu}$. For calculations of energy loss per unit length of a potassium ion in stainless steel, results of CRANGE and SRIM agree to within ten percent above $1.0 \mathrm{MeV} / \mathrm{amu}$. For calculations of the range of a helium ion in aluminum, results of CRANGE and ASTAR agree to within two percent above $1.0 \mathrm{MeV} / \mathrm{amu}$. Finally, for calculations of ion induced electron yield for hydrogen ions striking gold, results of CRANGE agree to within ten percent with measured electron yields above $1.0 \mathrm{MeV} / \mathrm{amu}$.

PACS numbers:
\end{abstract}

Keywords:

*pstoltz@txcorp.com 


\section{INTRODUCTION}

Researchers believe stray electrons may limit the performance of proposed heavy ion accelerators for fusion energy and high energy density physics applications [1, 2]. One source of stray electrons is electrons produced when beam ions strike the accelerator beam pipe walls. Modeling these electrons, therefore, requires accurately modeling ion-material interactions in the walls [3]. A standard code for this modeling is the SRIM code [4], but SRIM has two limitations: (i) SRIM runs only on the Windows platform and (ii) the source code for SRIM is difficult to obtain. The first limitation means interfacing SRIM with other codes is difficult because many codes run on the Linux platform. The second limitation means one cannot easily add new features to SRIM. PSTAR [5] and ASTAR [6] are other well-known codes for calculating ion energy loss and range in materials, but these codes are specific to protons and alpha particles.

Consequently, we have begun using the CRANGE code [7] for studying ion-material interactions involving heavy ions. Because CRANGE is open source, one can modify it and use it in combination with other codes. However, because CRANGE is not well known, we wanted to check the results against the better known codes and against data to ensure CRANGE produces reasonable results. In this paper, we present comparisons of ion energy loss and range calculated by CRANGE to SRIM and ASTAR. Also, we compare electron yield predicted with CRANGE to experimentally measured yields [8] for protons striking gold. For calculations of energy loss per unit length of a potassium ion in stainless steel, results of CRANGE and SRIM agree to within ten percent above $1.0 \mathrm{MeV} / \mathrm{amu}$. For calculations of the range of a helium ion in aluminum, results of CRANGE and ASTAR agree to within two percent above $1.0 \mathrm{MeV} / \mathrm{amu}$. Finally, results of CRANGE agree to within ten percent with measured electron yields above $1.0 \mathrm{MeV} / \mathrm{amu}$ for protons striking gold.

\section{BACKGROUND}

The CRANGE algorithms are based on the Bethe stopping formula, but include corrections for density, nuclear size, and other effects. The authors of CRANGE developed the code for high energy applications (above $1.0 \mathrm{MeV} / \mathrm{amu}$ ). Consequently, the algorithms model only electronic stopping of ions (nuclear stopping is neglected). Because of the present 


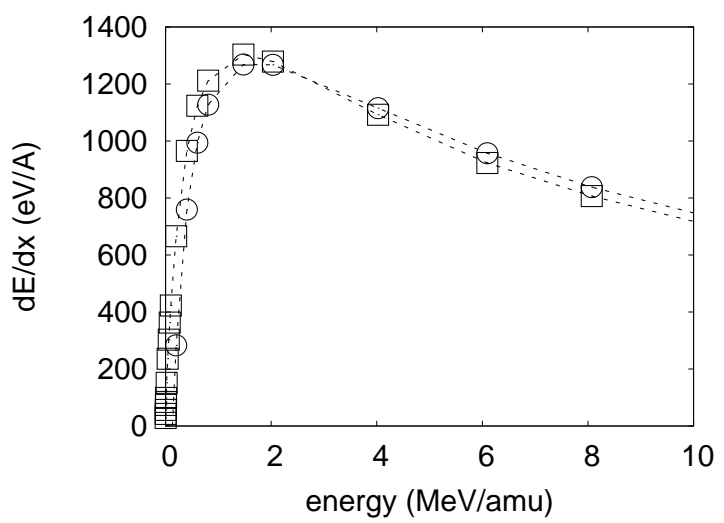

FIG. 1: Comparison of simulation results of CRANGE (circles) and SRIM (squares) of energy loss per unit length as a function of energy for potassium in stainless steel. Results agree to within a factor of two above $0.1 \mathrm{MeV} / \mathrm{amu}$ and to within a ten percent above $1.0 \mathrm{MeV} / \mathrm{amu}$.

limitation of the algorithms, we did not expect quantitative agreement for the comparisons of CRANGE to other codes at energies below 1.0 MeV/amu. We restrict ourselves mainly to comparisons above $1.0 \mathrm{MeV} / \mathrm{amu}$, where we know the CRANGE algorithms to be more accurate. Improving the CRANGE algorithms below $1.0 \mathrm{MeV} / \mathrm{amu}$ is something we plan for future work, as we discuss in the conclusions. For the comparisons shown here, we include the density effect correction in the CRANGE algorithms, but not any others (other corrections are typically important only at very high energies).

\section{COMPARISONS TO OTHER CODES AND EXPERIMENT}

We first compare CRANGE to SRIM for simulations of potassium ions stopping in stainless steel. These simulations are relevant to the High Current Experiment at Lawrence Berkeley National Laboratory [9]. Figure 1 shows simulation results for energy loss per unit length as a function of energy for both CRANGE and SRIM. Results agree to within ten percent above $1.0 \mathrm{MeV} / \mathrm{amu}$, the energy regime where we expect CRANGE to be most accurate. Below 1.0 MeV/amu, down to $0.1 \mathrm{MeV} / \mathrm{amu}$, results still agree to within a factor of two.

Next we compare CRANGE to ASTAR simulations of the range of alpha particles in aluminum. We chose aluminum merely as a contrast to stainless steel to test how CRANGE handles lighter target materials. Also, aluminum tests how CRANGE handles elemental 


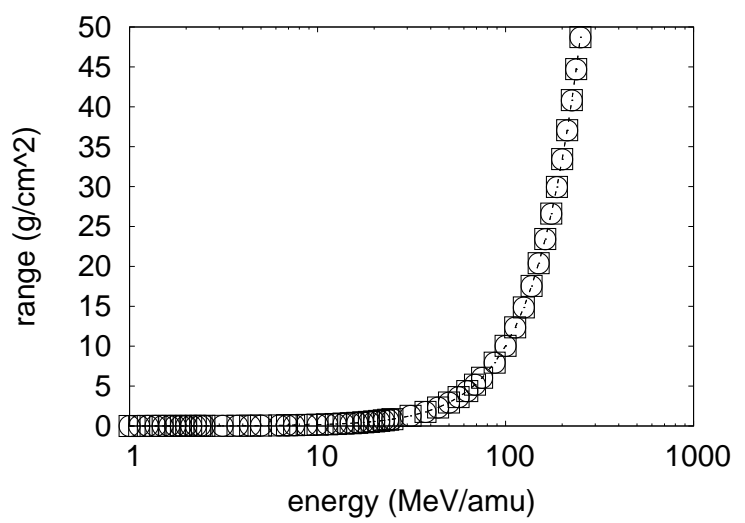

FIG. 2: Comparison of simulation results for CRANGE (circles) and ASTAR (squares) for the range of helium ions in aluminum by the function of energy. Results agree to within two percent above $1.0 \mathrm{MeV} / \mathrm{amu}$.

targets, as opposed to the previous test using stainless steel, a compound target. Figure 2 shows the range as a function of energy for CRANGE (circles) and ASTAR (squares). Results agree to within two percent above $1.0 \mathrm{MeV} / \mathrm{amu}$.

Finally, we compare CRANGE simulations of the electron yield for protons striking a gold surface to experimentally measured values. Figure 3 shows the electron yield calculated by CRANGE (circles) and experimentally measured (squares) as a function of energy. The CRANGE simulation results assume the electron yield is directly proportional to energy loss per unit length, so we calculate energy loss with CRANGE and normalize the results to agree with the experimental result at the highest energy (13.0 MeV/amu). As a check that this normalization is reasonable, it agrees to within 40 percent with the value suggested by the authors of Ref. [10]. The 40 percent agreement is reasonable because the value in Ref. [10] is an average value for all heavy ions and all targets.

\section{CONCLUSION}

The goal of this work was to gain confidence in the results of a new ion-material interaction code, CRANGE, by comparing it with other codes and with experiment. We compared the CRANGE code to SRIM and ASTAR, and to experimental results for effects such as energy loss per unit length, ion range, and ion induced electron yield. These types of simulations are relevant to heavy ions accelerators for fusion energy and high energy density physics. 


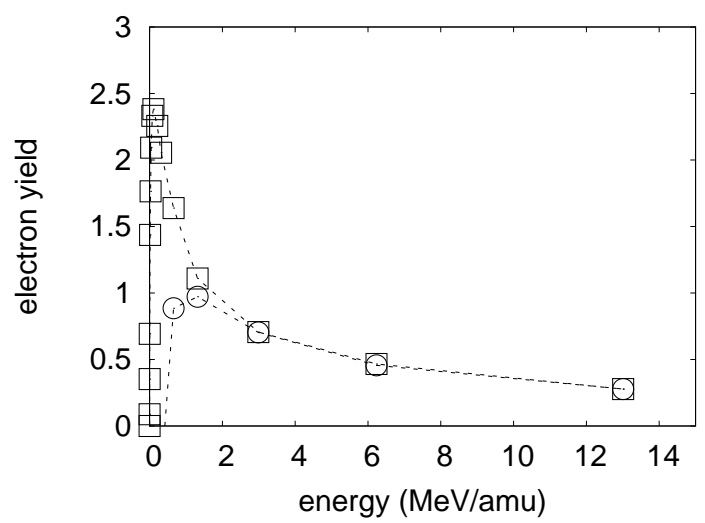

FIG. 3: Comparison of CRANGE (circles) simulated electron yield as a function of energy to measured values (squares) for protons striking gold. The CRANGE simulation results are results for energy loss per unit length, normalized to agree with the experimental result at the highest energy $(13.0 \mathrm{MeV} / \mathrm{amu})$. Results agree to within ten percent above $1.0 \mathrm{MeV} / \mathrm{amu}$.

For calculations of energy loss per unit length of a potassium ion in stainless steel, results of CRANGE and SRIM agree to within a factor of two above $0.1 \mathrm{MeV} / \mathrm{amu}$ and to within a ten percent above $1.0 \mathrm{MeV} / \mathrm{amu}$. For calculations of the range of a helium ion in aluminum, results of CRANGE and ASTAR agree to within two percent above $1.0 \mathrm{MeV} / \mathrm{amu}$. Finally, for calculations of ion induced electron yield for hydrogen ions striking gold, results of CRANGE agree to within ten percent with measured electron yields above $1.0 \mathrm{MeV} / \mathrm{amu}$.

We plan to modify the CRANGE code to be more accurate at lower energies (energies below $1.0 \mathrm{MeV} / \mathrm{amu}$ ). At lower energies, interactions between the ions and target nuclei become important. This includes a contribution to the energy loss and a deflection of the ion trajectory. Consequently, one modification we plan is to add nuclear stopping and angular scattering. Also, we plan to develop a user-friendly interface to CRANGE, with the capability to use CRANGE from the scripting language, Python [11] (we used a prototype of this Python interface in generating the plots shown in this paper).

\section{Acknowledgments}

The authors thank B. Weaver for help with the CRANGE code. This work was supported partially by the U.S. Department of Energy by Small Business Innovation Research grant DE-FG02-03ER83797. This work was performed under the auspices of the U.S. Department 
of Energy by Lawrence Livermore National Laboratory under contract W7405-ENG-48 and by Lawrence Berkeley National Laboratory under contract DE-AC03-76F00098.

[1] ECLOUD 02 Workshop, CERN Report CERN-2002-001, http:// wwwslap.cern.ch/collective/ecloud02/proceedings/

[2] ECLOUD 04 Workshop, http://icfa-ecloud04.web.cern.ch/icfa-ecloud04/agenda.html

[3] P. Stoltz, et al, Phys. Rev. ST Accel. Beams 6, 054701 (2003).

[4] J. F. Ziegler, J. P. Biersack, and U. Littmark, "The Stopping and Range of Ions in Solids", Pergamon Press, New York (1985)

[5] http://physics.nist.gov/PhysRefData/Star/Text/PSTAR.html

[6] http://physics.nist.gov/PhysRefData/Star/Text/ASTAR.html

[7] B. A. Weaver and A. J. Westphal, Nucl. Instrum. and Meth. B, 187 (2002) p. 285

[8] H. Eder, et al, Review of Scientific Instruments 68, 165 (1997).

[9] A. W. Molvik, et al, "Initial Experimental Studies of Electron Accumulation in a Heavy-Ion Beam", Particle Accelerator Conference, Portland OR, 2003, paper TOPC004; http://epaper.kek.jp/p03/INDEX.HTM

[10] H. Rothard, et al, Physical Review A 41, 2521 (1990).

[11] http://python.org 